University of Nebraska - Lincoln

DigitalCommons@University of Nebraska - Lincoln

USDA National Wildlife Research Center - Staff Publications
U.S. Department of Agriculture: Animal and Plant Health Inspection Service

2011

\title{
A review and synthesis of bird and rodent damage estimates to select California crops
}

\author{
Karen Gebhardt \\ USDA/APHIS/WS National Wildlife Research Center, karen.gebhardt@colorado.edu
}

Aaron M. Anderson

USDA/APHIS/WS National Wildlife Research Center, aaron.m.anderson@aphis.usda.gov

Katy N. Kirkpatrick

USDA/APHIS/WS National Wildlife Research Center, Katy.N.Kirkpatrick@aphis.usda.gov

Stephanie A. Shwiff

USDA/APHIS/WS National Wildlife Research Center, stephanie.a.shwiff@aphis.usda.gov

Follow this and additional works at: https://digitalcommons.unl.edu/icwdm_usdanwrc

Part of the Environmental Sciences Commons

Gebhardt, Karen; Anderson, Aaron M.; Kirkpatrick, Katy N.; and Shwiff, Stephanie A., "A review and synthesis of bird and rodent damage estimates to select California crops" (2011). USDA National Wildlife Research Center - Staff Publications. 1038.

https://digitalcommons.unl.edu/icwdm_usdanwrc/1038

This Article is brought to you for free and open access by the U.S. Department of Agriculture: Animal and Plant Health Inspection Service at DigitalCommons@University of Nebraska - Lincoln. It has been accepted for inclusion in USDA National Wildlife Research Center - Staff Publications by an authorized administrator of DigitalCommons@University of Nebraska - Lincoln. 
Review

\title{
A review and synthesis of bird and rodent damage estimates to select California crops
}

\author{
Karen Gebhardt $^{\text {a,b }}$, Aaron M. Anderson ${ }^{a}$, Katy N. Kirkpatrick ${ }^{a}$, Stephanie A. Shwiff ${ }^{a, *}$ \\ ${ }^{a}$ USDA/APHIS/WS National Wildlife Research Center, 4101 LaPorte Ave. Fort Collins, CO 80521, USA \\ ${ }^{\mathrm{b}}$ Department of Economics, Colorado State University, Fort Collins, CO 80523, USA
}

\section{A R T I C L E I N F O}

\section{Article history:}

Received 5 October 2010

Received in revised form

18 April 2011

Accepted 16 May 2011

\section{Keywords:}

California

Vertebrate pest

Pest damage

Bird

Rodent

Economics

\begin{abstract}
A B S T R A C T
A comprehensive literature review was conducted to identify the magnitude of bird and rodent damage to 19 economically important crops in California. Interviews with agriculture experts provided additional information about damages. Monte Carlo simulations were used to derive summary estimates of damages to each crop. A meta-analysis indicated that summary damage estimates from expert interviews were higher than estimates from field studies and surveys. It was also found that there has been a downward trend over time in damages to almonds and grapes. The results of our study indicate that damages from bird and rodents remain high for many crops and are likely to be economically significant within the state of California.
\end{abstract}

Published by Elsevier Ltd.

\section{Introduction}

The negative impacts (e.g., bird, rodent, and insect damage and drought) to agricultural production in California can have a major effect on the state's economy and consumers throughout the U.S. and around the world. Understanding the level of damage caused by birds and rodents is crucial to effective implementation of management strategies and techniques to mitigate the negative impact and thereby minimize the effect on the greater economy.

Damage caused by birds and rodents can be severe, diverse, and vary across time and geography. Examples include crows consuming grapes and almonds as well as ground squirrels girdling trees and feeding on alfalfa. Growers employ a variety of strategies and techniques to combat crop loss including the use of rodenticides and avicides, trapping, exclusion, and chemical aversion (Sexton et al., 2007). Although these techniques are generally effective at limiting crop loss, damage due to birds and rodents remains a problem in California.

Research on bird and rodent damage consists predominantly of individual studies on either a single species or multiple species

\footnotetext{
* Corresponding author. Tel.: +1970 266 6150; fax: +1970 2666157.

E-mail addresses: Karen.Gebhardt@colostate.edu (K. Gebhardt), Aaron.M. Anderson@aphis.usda.gov (A.M. Anderson), Katy.N.Kirkpatrick@aphis.usda.gov (K.N. Kirkpatrick), Stephanie.A.Shwiff@aphis.usda.gov (S.A. Shwiff).
}

impacting a single crop's final product (see Crase et al., 1976; Hothem et al., 1981; Gadd, 1996; Cummings et al., 2005; Berge et al., 2007; Delwiche et al., 2007) or a single species impacting multiple crops' final product (see DeHaven, 1974; Marsh, 1998). A shortcoming of these studies is their limited focus. A multi-crop, multi-region analysis would allow investigation of the broader impact that birds and rodents have on California agriculture and the California economy, and would allow a more comprehensive assessment of the benefits of employing various pest control methods.

A limited amount of research has attempted to incorporate multiple pest species' damage to multiple crops (see Razee, 1976; NASS, 1999; NASS, 2002; Hueth et al., 1997). One of the most comprehensive studies was Hueth et al. (1997), which undertook an analysis of the economic impact of vertebrate pest damage to select California crops. Although the study was a multi-crop, multiregion analysis, the estimates of damage were obtained from a very limited number of interviews and published studies.

Our study builds on previous research by compiling the estimates of bird and rodent damage to 19 economically important crops in select regions within California. The crops studied were alfalfa, almonds, artichokes, broccoli, carrots, cherries, grapes, lettuce, lemons, melons, nursery products, oranges, pistachios, peaches, rice, spinach, strawberries, tomatoes, and walnuts. Damage estimates were gathered from several types of sources, including previously published estimates, unpublished studies, and interviews with 
Table 1

Review of bird and rodent damage estimates and sources.

\begin{tabular}{|c|c|c|c|c|c|c|c|}
\hline \multicolumn{3}{|c|}{ Damage per Acre (\%) } & \multirow[t]{2}{*}{ Pest } & \multirow[t]{2}{*}{ Region } & \multirow[t]{2}{*}{ Year of Study } & \multirow[t]{2}{*}{ Type } & \multirow[t]{2}{*}{ Source } \\
\hline Low & Mid & High & & & & & \\
\hline \multicolumn{8}{|c|}{ Almonds } \\
\hline 3 & 4 & 15 & Vertebrate pests & California & 2008 & Interview & Marsh, 2008 \\
\hline 1.52 & 2.07 & 5.05 & Vertebrate pests & Fresno County & 2008 & Interview & Sagardia and Sagardia, 2008 \\
\hline- & 15 & - & Vertebrate pests & Fresno County & 2008 & Interview & Taber, 2008 \\
\hline- & 0.0065 & - & Birds, crows & Fresno County & 2003 & Field Study & Delwiche et al., 2007 \\
\hline- & 0.0335 & - & Birds, crows & Fresno County & 2003 & Field Study & Delwiche et al., 2007 \\
\hline- & 0.33525 & - & Birds, crows & Yuba County & 2003 & Field Study & Delwiche et al., 2007 \\
\hline- & 0.004 & - & Birds, crows & Fresno County & 2003 & Field Study & Delwiche et al., 2007 \\
\hline- & 0.058 & - & Birds, crows & Fresno County & 2003 & Field Study & Delwiche et al., 2007 \\
\hline- & 0.0315 & - & Birds, crows & Yuba County & 2003 & Field Study & Delwiche et al., 2007 \\
\hline- & 0.0065 & - & Birds, crows & Fresno County & 2002 & Field Study & Delwiche et al., 2007 \\
\hline- & 0.242 & - & Birds, crows & Fresno County & 2002 & Field Study & Delwiche et al., 2007 \\
\hline- & 0.94 & - & Birds, crows & Yuba County & 2002 & Field Study & Delwiche et al., 2007 \\
\hline- & 0.006 & - & Birds, crows & Fresno County & 2002 & Field Study & Delwiche et al., 2007 \\
\hline- & 0.0755 & - & Birds, crows & Fresno County & 2002 & Field Study & Delwiche et al., 2007 \\
\hline- & 0.06675 & - & Birds, crows & Yuba County & 2002 & Field Study & Delwiche et al., 2007 \\
\hline- & 0.0989 & - & Crows & Sacramento Valley & 1999 & Field Study & Salmon et al., 2000 \\
\hline- & 2.03 & - & Crows & Sacramento Valley & 1999 & Field Study & Salmon et al., 2000 \\
\hline- & 0.0465 & - & Crows & Sacramento Valley & 1999 & Field Study & Salmon et al., 2000 \\
\hline- & 0.0407 & - & Crows & Sacramento Valley & 1999 & Field Study & Salmon et al., 2000 \\
\hline- & 0.023 & - & Crows & Sacramento Valley & 1999 & Field Study & Salmon et al., 2000 \\
\hline- & 0.71 & - & Crows & San Joaquin Valley & 1999 & Field Study & Salmon et al., 2000 \\
\hline- & 7.05 & - & Crows & Sacramento Valley & 1999 & Field Study & Salmon et al., 2000 \\
\hline- & 0.97 & - & Crow, Magpie, Scrub Jay & Sacramento Valley & 1998 & Field Study & Salmon et al., 1999 \\
\hline- & 1.39 & - & Crow, Magpie, Scrub Jay & Sacramento Valley & 1998 & Field Study & Salmon et al., 1999 \\
\hline- & 6.1 & - & Crow, Magpie, Scrub Jay & Sacramento Valley & 1998 & Field Study & Salmon et al., 1999 \\
\hline- & 2.44 & - & Crow, Magpie, Scrub Jay & Sacramento Valley & 1998 & Field Study & Salmon et al., 1999 \\
\hline- & 0.128 & - & Crows & Sacramento Valley & 1998 & Field Study & Salmon et al., 1999 \\
\hline 0.03 & - & 0.04 & Deer mice & Central Valley and Sacramento Valley & 1997-99 & Field Study & Pearson et al., 2000 \\
\hline 0.07 & - & 0.10 & Deer mice & Central Valley and Sacramento Valley & 1997-99 & Field Study & Pearson et al., 2000 \\
\hline 0.10 & - & 0.16 & Birds, crows, magpies & Central Valley and Sacramento Valley & 1997-99 & Field Study & Pearson et al., 2000 \\
\hline 0.06 & - & 0.09 & Western gray squirrel & Central Valley and Sacramento Valley & 1997-99 & Field Study & Pearson et al., 2000 \\
\hline- & 2.34 & - & Crows & Yolo County & 1997 & Field Study & Salmon et al., 1997 \\
\hline- & 1.32 & - & Crows & Yolo County & 1997 & Field Study & Salmon et al., 1997 \\
\hline- & 29.53 & - & Crows & Sutter County & 1997 & Field Study & Salmon et al., 1997 \\
\hline- & 10.57 & - & Crows & Sutter County & 1997 & Field Study & Salmon et al., 1997 \\
\hline- & 4.22 & - & Crows & Sutter County & 1997 & Field Study & Salmon et al., 1997 \\
\hline- & 3.5 & - & Vertebrate pests & California & $1996-97$ & Mixed & Hueth et al., 1997 \\
\hline 3 & & 4 & Crows & Yuba and Sutter Counties & 1988 & Field Study & Hasey and Salmon, 1993 \\
\hline- & 4.1 & - & Crow, Scrub Jay, Magpie & Merced County & 1984 & Survey & CDFA, 1984 \\
\hline- & 3 & - & Crow, Scrub Jay, Magpie & San Joaquin County & 1984 & Survey & CDFA, 1984 \\
\hline- & 1.5 & - & Crow, Scrub Jay, Magpie & Butte County & 1984 & Survey & CDFA, 1984 \\
\hline- & 30 & - & Crow, Scrub Jay, Magpie & Fresno County & 1984 & Survey & CDFA, 1984 \\
\hline- & 6 & - & Crow, Scrub Jay, Magpie & Colusa County & 1984 & Survey & CDFA, 1984 \\
\hline- & 1 & - & Crow, Scrub Jay, Magpie & Kings County & 1984 & Survey & CDFA 1984 \\
\hline- & 1.8 & - & Crow, Scrub Jay, Magpie & Glenn County & 1984 & Survey & CDFA, 1984 \\
\hline- & 16 & - & Crow, Scrub Jay, Magpie & Tulare County & 1984 & Survey & CDFA 1984 \\
\hline- & 0.12 & - & Crow, Scrub Jay, Magpie & Solano County & 1984 & Survey & CDFA, 1984 \\
\hline- & 5 & - & Crow, Scrub Jay, Magpie & Contra Costa County & 1984 & Survey & CDFA, 1984 \\
\hline 6 & - & 18 & Crows & Tulare County & 1966 & Interview & Simpson, 1972 \\
\hline- & 7 & - & Birds, linnets, crows, jays, etc. & Sacramento Valley & $1935-36$ & Field Study & Emlen, 1937 \\
\hline- & 21 & - & Birds, linnets, crows, jays, etc. & Sacramento Valley & $1935-36$ & Field Study & Emlen, 1937 \\
\hline- & 28 & - & Birds, linnets, crows, jays, etc. & Sacramento Valley & $1935-36$ & Field Study & Emlen, 1937 \\
\hline \multicolumn{8}{|c|}{ Artichokes } \\
\hline 20 & - & 30 & Voles, gophers & California & 2008 & Interview & Roach, 2008 \\
\hline 1.5 & 3 & - & Vertebrate pests & California & 2008 & Interview & Marsh, 2008 \\
\hline- & 15 & - & Vertebrate pests & California & 1996-97 & Mixed & Hueth et al., 1997 \\
\hline \multicolumn{8}{|c|}{ Broccoli } \\
\hline- & 0.6 & - & Birds and rodents & Fresno County & 2008 & Interview & Strmiska, 2008 \\
\hline- & 0.1 & - & Vertebrate pests & California & 2008 & Interview & Marsh, 2008 \\
\hline- & 100 & - & Ground squirrels & Santa Cruz County & $2003-04$ & Field Study & Muramoto et al., 2005 \\
\hline \multicolumn{8}{|c|}{ Carrots } \\
\hline- & 0.1 & - & Vertebrate pests & California & 2008 & Interview & Marsh, 2008 \\
\hline- & 0.62 & - & Vertebrate pests & California & 1996-97 & Mixed & Hueth et al., 1997 \\
\hline Cherr & & & & & & & \\
\hline 5 & - & 6 & Vertebrate pests & California & 2008 & Interview & Marsh, 2008 \\
\hline- & - & 50 & Birds & Fresno County & 2008 & Interview & Taber, 2008 \\
\hline 7.62 & - & 10 & Birds & California & $1975-76$ & Field Study & DeHaven et al., 1979 \\
\hline
\end{tabular}


Table 1 (continued)

\begin{tabular}{|c|c|c|c|c|c|c|c|}
\hline \multicolumn{3}{|c|}{ Damage per Acre (\%) } & \multirow[t]{2}{*}{ Pest } & \multirow[t]{2}{*}{ Region } & \multirow[t]{2}{*}{ Year of Study } & \multirow[t]{2}{*}{ Type } & \multirow[t]{2}{*}{ Source } \\
\hline Low & Mid & High & & & & & \\
\hline \multicolumn{8}{|c|}{ Citrus } \\
\hline- & 3.5 & - & Vertebrate pests & California & 1996-97 & Mixed & Hueth et al., 1997 \\
\hline- & 0.5 & - & Vertebrate pests & California & 1996-97 & Mixed & Hueth et al., 1997 \\
\hline \multicolumn{8}{|c|}{ Table Grapes } \\
\hline 0.76 & - & 0.95 & Birds & Fresno County & 2008 & Interview & Pitts, 2008 \\
\hline 0.07 & - & 0.14 & Rodents & Fresno County & 2008 & Interview & Pitts, 2008 \\
\hline- & 25 & 35 & Birds & Fresno County & 2008 & Interview & Vasquez, 2008 \\
\hline 0.5 & - & 1 & Rodents & Fresno County & 2008 & Interview & Vasquez, 2008 \\
\hline- & 0.87 & - & Wildlife damage & California & 1998 & Survey & NASS, 1999 \\
\hline- & 3.5 & - & Vertebrate pests & California & 1996-97 & Mixed & Hueth et al., 1997 \\
\hline 0.43 & - & 0.71 & Birds & California & 1976 & Survey & Razee, 1976 \\
\hline- & 1 & - & Birds & California & 1973 & Survey & Stone, 1973 \\
\hline 0.1 & 9.6 & 30 & Birds & California & 1973 & Survey & Crase et al., 1976 \\
\hline \multicolumn{8}{|c|}{ Wine Grapes } \\
\hline- & 25 & 35 & Birds & Fresno County & 2008 & Interview & Vasquez, 2008 \\
\hline 0.5 & - & 1 & Rodents & Fresno County & 2008 & Interview & Vasquez, 2008 \\
\hline- & 3 & 35 & Birds & Napa County & 2008 & Interview & Goymerac, 2008 \\
\hline 3 & 5 & - & Birds & Napa County & 2008 & Interview & Goymerac, 2008 \\
\hline 0.5 & - & 2 & Birds & Fresno and Napa Counties & 2008 & Interview & Taber, 2008 \\
\hline 50 & - & 60 & Birds & Fresno and Napa Counties & 2008 & Interview & Taber, 2008 \\
\hline 1 & 13 & 20 & Birds & Napa County & 2008 & Interview & Witmer, 2008 \\
\hline- & 11.1 & - & Birds & Napa and Sonoma Counties & 2005 & Field Study & Berge et al., 2007 \\
\hline- & 14.9 & - & Birds & Napa and Sonoma Counties & 2005 & Field Study & Berge et al., 2007 \\
\hline- & 7.7 & - & Birds & Napa and Sonoma Counties & 2005 & Field Study & Berge et al., 2007 \\
\hline- & 2.8 & - & Birds & Napa and Sonoma Counties & 2005 & Field Study & Berge et al., 2007 \\
\hline- & 6.5 & - & Birds & Napa and Sonoma Counties & 2005 & Field Study & Berge et al., 2007 \\
\hline- & 3.8 & - & Birds & Napa and Sonoma Counties & 2005 & Field Study & Berge et al., 2007 \\
\hline- & 0.7 & - & Birds & Napa and Sonoma Counties & 2005 & Field Study & Berge et al., 2007 \\
\hline- & 7.7 & - & Birds & Napa and Sonoma Counties & 2004 & Field Study & Berge et al., 2007 \\
\hline- & 11.6 & - & Birds & Napa and Sonoma Counties & 2004 & Field Study & Berge et al., 2007 \\
\hline- & 5.3 & - & Birds & Napa and Sonoma Counties & 2004 & Field Study & Berge et al., 2007 \\
\hline- & 8.5 & - & Birds & Napa and Sonoma Counties & 2004 & Field Study & Berge et al., 2007 \\
\hline- & 8.4 & - & Birds & Napa and Sonoma Counties & 2004 & Field Study & Berge et al., 2007 \\
\hline- & 2 & - & Birds & Napa and Sonoma Counties & 2004 & Field Study & Berge et al., 2007 \\
\hline- & 1.2 & - & Birds & Napa and Sonoma Counties & 2004 & Field Study & Berge et al., 2007 \\
\hline- & 0.5 & - & Birds & Napa and Sonoma Counties & 2004 & Field Study & Berge et al., 2007 \\
\hline- & 0.87 & - & Wildlife damage & California & 1998 & Survey & NASS, 1999 \\
\hline- & 1.02 & - & Vertebrate pests & California & $1996-97$ & Mixed & Hueth et al., 1997 \\
\hline- & 11 & - & House finch & Sonoma County & 1996 & Field Study & Gadd, 1996. \\
\hline- & 2.5 & - & $\begin{array}{l}\text { Robin, house finches, } \\
\text { quail, and goldfinches }\end{array}$ & Napa and Sonoma Counties & 1978 & Field Study & Hothem et al., 1981 \\
\hline- & 1.5 & - & $\begin{array}{l}\text { Robin, house finches, } \\
\text { quail, and goldfinches }\end{array}$ & Napa and Sonoma Counties & 1978 & Field Study & Hothem et al., 1981 \\
\hline- & 4.75 & - & $\begin{array}{l}\text { Robin, house finches, } \\
\text { quail, and goldfinches }\end{array}$ & Napa and Sonoma Counties & 1978 & Field Study & Hothem et al., 1981 \\
\hline- & 2 & - & $\begin{array}{l}\text { Robin, house finches, } \\
\text { quail, and goldfinches }\end{array}$ & Napa and Sonoma Counties & 1978 & Field Study & Hothem et al., 1981 \\
\hline- & 2.5 & - & $\begin{array}{l}\text { Robin, house finches, } \\
\text { quail, and goldfinches }\end{array}$ & Napa and Sonoma Counties & 1978 & Field Study & Hothem et al., 1981 \\
\hline- & 2 & - & $\begin{array}{l}\text { Robin, house finches, } \\
\text { quail, and goldfinches }\end{array}$ & Napa and Sonoma Counties & 1978 & Field Study & Hothem et al., 1981 \\
\hline- & 1.25 & - & $\begin{array}{l}\text { Robin, house finches, } \\
\text { quail, and goldfinches }\end{array}$ & Napa and Sonoma Counties & 1978 & Field Study & Hothem et al., 1981 \\
\hline- & 1 & - & $\begin{array}{l}\text { Robin, house finches, } \\
\text { quail, and goldfinches }\end{array}$ & Napa and Sonoma Counties & 1978 & Field Study & Hothem et al., 1981 \\
\hline- & 7.75 & - & $\begin{array}{l}\text { Robin, house finches, } \\
\text { quail, and goldfinches }\end{array}$ & Napa and Sonoma Counties & 1978 & Field Study & Hothem et al., 1981 \\
\hline- & 5.5 & - & $\begin{array}{l}\text { Robin, house finches, } \\
\text { quail, and goldfinches }\end{array}$ & Napa and Sonoma Counties & 1978 & Field Study & Hothem et al., 1981 \\
\hline- & 3.25 & - & $\begin{array}{l}\text { Robin, house finches, } \\
\text { quail, and goldfinches }\end{array}$ & Napa and Sonoma Counties & 1978 & Field Study & Hothem et al., 1981 \\
\hline- & 13.3 & - & $\begin{array}{l}\text { Robin, house finches, } \\
\text { quail, and goldfinches }\end{array}$ & Napa and Sonoma Counties & 1978 & Field Study & Hothem et al., 1981 \\
\hline- & 7.75 & - & $\begin{array}{l}\text { Robin, house finches, } \\
\text { quail, and goldfinches }\end{array}$ & Napa and Sonoma Counties & 1978 & Field Study & Hothem et al., 1981 \\
\hline- & 4 & - & $\begin{array}{l}\text { Robin, house finches, } \\
\text { quail, and goldfinches }\end{array}$ & Napa and Sonoma Counties & 1978 & Field Study & Hothem et al., 1981 \\
\hline- & 2.5 & - & $\begin{array}{l}\text { Robin, house finches, } \\
\text { quail, and goldfinches }\end{array}$ & Napa and Sonoma Counties & 1978 & Field Study & Hothem et al., 1981 \\
\hline- & 2.5 & - & $\begin{array}{l}\text { Robin, house finches, } \\
\text { quail, and goldfinches }\end{array}$ & Napa and Sonoma Counties & 1978 & Field Study & Hothem et al., 1981 \\
\hline
\end{tabular}


Table 1 (continued)

\begin{tabular}{|c|c|c|c|c|c|c|c|}
\hline \multicolumn{3}{|c|}{ Damage per Acre (\%) } & \multirow[t]{2}{*}{ Pest } & \multirow[t]{2}{*}{ Region } & \multirow[t]{2}{*}{ Year of Study } & \multirow[t]{2}{*}{ Type } & \multirow[t]{2}{*}{ Source } \\
\hline Low & Mid & High & & & & & \\
\hline- & 1.4 & - & $\begin{array}{l}\text { House finches, } \\
\text { quail, and robins }\end{array}$ & $\begin{array}{l}\text { Napa, Sonoma, and San } \\
\text { Joaquin Counties }\end{array}$ & $1977-78$ & Field Study & DeHaven and Hothem, 1980 \\
\hline- & 2.6 & - & $\begin{array}{l}\text { House finches, } \\
\text { quail, and robins }\end{array}$ & $\begin{array}{l}\text { Napa, Sonoma, and San } \\
\text { Joaquin Counties }\end{array}$ & $1977-78$ & Field Study & DeHaven and Hothem, 1980 \\
\hline- & 6.3 & - & $\begin{array}{l}\text { House finches, } \\
\text { quail, and robins }\end{array}$ & $\begin{array}{l}\text { Napa, Sonoma, and San } \\
\text { Joaquin Counties }\end{array}$ & $1977-78$ & Field Study & DeHaven and Hothem, 1980 \\
\hline- & 9.8 & - & $\begin{array}{l}\text { House finches, } \\
\text { quail, and robins }\end{array}$ & $\begin{array}{l}\text { Napa, Sonoma, and San } \\
\text { Joaquin Counties }\end{array}$ & $1977-78$ & Field Study & DeHaven and Hothem, 1980 \\
\hline- & 15.1 & - & $\begin{array}{l}\text { House finches, } \\
\text { quail, and robins }\end{array}$ & $\begin{array}{l}\text { Napa, Sonoma, and San } \\
\text { Joaquin Counties }\end{array}$ & $1977-78$ & Field Study & DeHaven and Hothem 1980 \\
\hline- & 76.8 & - & $\begin{array}{l}\text { House finches, } \\
\text { quail, and robins }\end{array}$ & $\begin{array}{l}\text { Napa, Sonoma, and San } \\
\text { Joaquin Counties }\end{array}$ & $1977-78$ & Field Study & DeHaven and Hothem, 1980 \\
\hline 0.43 & - & 0.71 & Birds & California & 1976 & Survey & Razee, 1976 \\
\hline- & 1 & - & Birds & California & 1973 & Survey & Stone, 1973 \\
\hline 0.1 & 9.6 & 30 & Birds & California & 1973 & Survey & Crase et al., 1976. \\
\hline- & 10 & - & Birds & Alameda County & 1973 & Survey & DeHaven, 1974 \\
\hline- & 3.7 & - & Birds & Mendocino County & 1973 & Survey & DeHaven, 1974 \\
\hline- & 11.4 & - & Birds & Monterey County & 1973 & Survey & DeHaven, 1974 \\
\hline- & 16.9 & - & Birds & Napa County & 1973 & Survey & DeHaven 1974 \\
\hline- & 17.8 & - & Birds & San Benito County & 1973 & Survey & DeHaven, 1974 \\
\hline- & 54.7 & - & Birds & Santa Clara County & 1973 & Survey & DeHaven, 1974 \\
\hline- & 11.7 & - & Birds & Solano County & 1973 & Survey & DeHaven, 1974 \\
\hline- & 14.7 & - & Birds & Sonoma County & 1973 & Survey & DeHaven, 1974 \\
\hline \multicolumn{8}{|c|}{ Alfalfa } \\
\hline- & 7.6 & - & Belding's ground squirrel & Surprise Valley & 1999 & Field Study & Whisson et al., 2000 \\
\hline- & 6.97 & - & Belding's ground squirrel & Butte Valley & 1999 & Field Study & Whisson et al., 2000 \\
\hline- & 9.5 & - & Belding's ground squirrel & Butte Valley & 1998 & Field Study & Whisson et al., 2000 \\
\hline- & 10.76 & - & Belding's ground squirrel & Surprise Valley & 1998 & Field Study & Whisson et al., 2000 \\
\hline- & 7.6 & - & Belding's ground squirrel & Butte Valley & 1997 & Field Study & Whisson et al., 2000 \\
\hline- & 7.83 & - & Vertebrate pests & California & 1996-97 & Field Study & Hueth et al., 1997 \\
\hline- & 37 & - & Belding's ground squirrel & Siskiyou County, Butte Valley & 1996 & Field Study & Whisson et al., 1999 \\
\hline- & 45.9 & - & Belding's ground squirrel & Siskiyou County, Butte Valley & 1996 & Field Study & Whisson et al., 1999 \\
\hline- & 34.6 & - & Belding's ground squirrel & Siskiyou County, Butte Valley & 1996 & Field Study & Whisson et al., 1999 \\
\hline- & 18.3 & - & Belding's ground squirrel & Siskiyou and Modoc Counties & 1995 & Field Study & Whisson et al., 1999 \\
\hline- & 48 & - & Belding's ground squirrel & Siskiyou and Modoc Counties & 1995 & Field Study & Whisson et al., 1999 \\
\hline- & 36.1 & - & Belding's ground squirrel & South central Oregon & 1977 & Field Study & Kalinowski and deCalesta, 1981 \\
\hline- & 53.8 & - & Belding's ground squirrel & South central Oregon & 1977 & Field Study & Kalinowski and deCalesta, 1981 \\
\hline- & 42.8 & - & Belding's ground squirrel & South central Oregon & 1977 & Field Study & Kalinowski and deCalesta, 1981 \\
\hline- & 40 & - & Belding's ground squirrel & South central Oregon & 1977 & Field Study & Kalinowski and deCalesta, 1981 \\
\hline- & 28.8 & - & Belding's ground squirrel & South central Oregon & 1977 & Field Study & Kalinowski and deCalesta, 1981 \\
\hline- & 28.8 & - & Belding's ground squirrel & South central Oregon & 1977 & Field Study & Kalinowski and deCalesta, 1981 \\
\hline- & 29.8 & - & Belding's ground squirrel & South central Oregon & 1977 & Field Study & Kalinowski and deCalesta, 1981 \\
\hline- & 17.6 & - & Belding's ground squirrel & South central Oregon & 1977 & Field Study & Kalinowski and deCalesta, 1981 \\
\hline- & 17.9 & - & Belding's ground squirrel & South central Oregon & 1977 & Field Study & Kalinowski and deCalesta, 1981 \\
\hline- & 17.6 & - & Belding's ground squirrel & South central Oregon & 1977 & Field Study & Kalinowski and deCalesta, 1981 \\
\hline- & 20.3 & - & Belding's ground squirrel & South central Oregon & 1977 & Field Study & Kalinowski and deCalesta, 1981 \\
\hline- & 17.5 & - & Belding's ground squirrel & Siskiyou and Modoc Counties & $1975-78$ & Field Study & Sauer, 1984 \\
\hline- & 28.4 & - & Belding's ground squirrel & Siskiyou and Modoc Counties & $1975-78$ & Field Study & Sauer, 1984 \\
\hline- & 19.5 & - & Belding's ground squirrel & Siskiyou and Modoc Counties & $1975-78$ & Field Study & Sauer, 1984 \\
\hline- & 21.1 & - & Belding's ground squirrel & Siskiyou and Modoc Counties & $1975-78$ & Field Study & Sauer, 1984 \\
\hline- & 19.5 & - & Belding's ground squirrel & Siskiyou and Modoc Counties & $1975-78$ & Field Study & Sauer, 1984 \\
\hline- & 38.5 & - & Belding's ground squirrel & Siskiyou and Modoc Counties & $1975-78$ & Field Study & Sauer, 1984 \\
\hline- & 17.1 & - & Belding's ground squirrel & Siskiyou and Modoc Counties & $1975-78$ & Field Study & Sauer, 1984 \\
\hline \multicolumn{8}{|c|}{ Lettuce } \\
\hline- & 1 & - & Birds & $\begin{array}{l}\text { Santa Cruz and Monterey } \\
\text { Counties }\end{array}$ & 2008 & Interview & Bolda, 2008 \\
\hline 3 & - & 4 & Birds & Fresno County & 2008 & Interview & Maya, 2008 \\
\hline 0 & - & - & Rodents & Fresno County & 2008 & Interview & Maya, 2008 \\
\hline- & - & 50 & Birds & Fresno County & 2008 & Interview & Maya, 2008 \\
\hline 1 & - & 2 & Rodents & Fresno County & 2008 & Interview & Maya, 2008 \\
\hline 30 & - & 100 & Birds and rodents & Fresno County & 2008 & Interview & Strmiska, 2008 \\
\hline- & 20 & - & Rodents & Fresno County & 2008 & Interview & Strmiska, 2008 \\
\hline 2 & - & 3 & Vertebrate pests & California & 2008 & Interview & Marsh 2008 \\
\hline- & 30 & - & Birds & Fresno County & 2008 & Interview & Taber, 2008 \\
\hline- & 0.6 & - & Horned lark & San Joaquin Valley & 1999 & Field Study & York et al., 2000 \\
\hline- & 3.75 & - & Vertebrate pests & California & $1996-97$ & Mixed & Hueth et al., 1997 \\
\hline \multicolumn{8}{|c|}{ Melon } \\
\hline 10 & - & 20 & Rodents & Fresno County & 2008 & Interview & Strmiska, 2008 \\
\hline- & 1 & - & Rodents & Fresno County & 2008 & Interview & Strmiska, 2008 \\
\hline- & 1.38 & - & Vertebrate pests & California & 2008 & Interview & Marsh, 2008 \\
\hline- & 0.1 & - & Birds & Fresno County & 2008 & Interview & Taber, 2008 \\
\hline- & 1.38 & - & Vertebrate pests & California & 1996-97 & Mixed & Hueth et al., 1997 \\
\hline
\end{tabular}


Table 1 (continued)

\begin{tabular}{|c|c|c|c|c|c|c|c|}
\hline \multicolumn{3}{|c|}{ Damage per Acre (\%) } & \multirow[t]{2}{*}{ Pest } & \multirow[t]{2}{*}{ Region } & \multirow[t]{2}{*}{ Year of Study } & \multirow[t]{2}{*}{ Type } & \multirow[t]{2}{*}{ Source } \\
\hline Low & Mid & High & & & & & \\
\hline \multicolumn{8}{|c|}{ Nursery } \\
\hline- & - & 5 & Cottontail rabbit & $\begin{array}{l}\text { Orange, San Diego, } \\
\text { and Los Angeles Counties }\end{array}$ & 2008 & Interview & Wilen, 2008 \\
\hline 4 & - & 6 & Cottontail rabbit & $\begin{array}{l}\text { Orange, San Diego, } \\
\text { and Los Angeles Counties }\end{array}$ & 2008 & Interview & Wilen, 2008 \\
\hline \multicolumn{8}{|c|}{ Peaches } \\
\hline- & 0.1 & - & Birds & Fresno County & 2008 & Interview & Taber, 2008 \\
\hline 1 & - & 2 & Vertebrate pests & California & 2008 & Interview & Marsh, 2008 \\
\hline 3 & - & 4 & Vertebrate pests & California & 2008 & Interview & Marsh, 2008 \\
\hline- & 0.68 & - & Vertebrate pests & California & 1996-97 & Mixed & Hueth et al., 1997 \\
\hline \multicolumn{8}{|c|}{ Pistachio } \\
\hline 3 & 4 & 15 & Vertebrate pests & California & 2008 & Interview & Marsh, 2008 \\
\hline- & 15 & - & Birds & Fresno County & 2008 & Interview & Taber, 2008 \\
\hline- & 5.75 & - & Vertebrate pests & California & 1996-97 & Mixed & Hueth et al., 1997 \\
\hline- & 0.91 & - & Crows & California & 1993 & Field Study & Hasey and Salmon, 1993 \\
\hline- & 4 & - & Birds & Tulare County & 1985 & Field Study & Crabb et al., 1986 \\
\hline- & 7.87 & - & Birds & Tulare County & 1985 & Field Study & Crabb et al., 1986 \\
\hline- & 12.2 & - & Birds & Tulare County & 1985 & Field Study & Crabb et al., 1986 \\
\hline 2 & 4 & 10 & $\begin{array}{l}\text { Crow, Raven, Jay, } \\
\text { Starling, Magpies }\end{array}$ & California & 1984 & Field Study & Crabb et al., 1986 \\
\hline- & 24 & - & $\begin{array}{l}\text { Crow, Scrub Jay, } \\
\text { Magpie }\end{array}$ & Tulare County & 1984 & Survey & CDFA, 1984 \\
\hline \multicolumn{8}{|l|}{ Rice } \\
\hline- & 1 & - & Blackbirds & California & 2001 & Field Study & Cummings et al., 2005 \\
\hline 0.1 & 0.2 & 3 & Birds & Sacramento Valley & 1972 & Survey & Stone, 1973 \\
\hline- & 0.1 & - & Birds & Sacramento Valley & 1971 & Interview & DeHaven, 1971 \\
\hline \multicolumn{8}{|c|}{ Rice (Wild) } \\
\hline 1 & - & 10 & Blackbirds & Sacramento Valley & 1993 & Survey & $\begin{array}{l}\text { Marcum and } \\
\text { Gorenzel, } 1994\end{array}$ \\
\hline \multicolumn{8}{|c|}{ Strawberry } \\
\hline- & 0.1 & - & Vertebrate pests & $\begin{array}{l}\text { Santa Cruz and } \\
\text { Monterey Counties }\end{array}$ & 2008 & Interview & Bolda, 2008 \\
\hline- & 0.1 & 10 & Vertebrate pests & $\begin{array}{l}\text { Santa Cruz and } \\
\text { Monterey Counties }\end{array}$ & 2008 & Interview & Moinar, 2008 \\
\hline- & 1.28 & - & Vertebrate pests & California & 1996-97 & Mixed & Hueth et al., 1997 \\
\hline \multicolumn{8}{|c|}{ Tomatoes } \\
\hline 0.1 & 1 & - & Vertebrate pests & California & 2008 & Interview & Marsh, 2008 \\
\hline- & 1.38 & - & Vertebrate pests & California & 1996-97 & Mixed & Hueth et al., 1997 \\
\hline- & 0.5 & & Vertebrate pests & California & 1996-97 & Mixed & Hueth et al., 1997 \\
\hline \multicolumn{8}{|c|}{ Walnuts } \\
\hline- & 0 & - & Vertebrate pests & California & 2008 & Interview & Marsh, 2008 \\
\hline- & 3 & 5 & Birds & Fresno County & 2008 & Interview & Taber, 2008 \\
\hline- & 2.8 & - & Vertebrate pests & California & 1996-97 & Mixed & Hueth et al., 1997 \\
\hline- & 4 & - & Crow, Scrub Jay, Magpie & Tulare County & 1984 & Survey & CDFA, 1984 \\
\hline- & 0.9 & - & Crow, Scrub Jay, Magpie & Butte County & 1984 & Survey & CDFA, 1984 \\
\hline- & 6 & - & Crow, Scrub Jay, Magpie & Merced County & 1984 & Survey & CDFA, 1984 \\
\hline 6 & - & 18 & Crows & Tulare County & 1966 & Interview & Simpson, 1972 \\
\hline
\end{tabular}

experts. A limited meta-analysis was performed to examine the impact that the data source has on the reported level of damage and examine any trend in damage over time. These damage estimates are reported by crop, and summary estimates based on the results of a Monte Carlo simulation of damages are then provided.

\section{Materials and methods}

To compile the bird and rodent damage data, a comprehensive search for published studies, surveys, and unpublished reports was conducted. Additional estimates were gathered in 2007 through personal interviews of agricultural extension specialists, County Agricultural Commissioners, crop growers, and knowledgeable wildlife damage specialists from across California. These experts were asked to estimate the amount of damage per acre caused by birds and rodents in either percent or monetary terms using current control methods for a particular crop. If estimates were given in monetary terms, they were converted to percent yield loss based on 3-year average price and yield data from California. In general, experts identified one or two major species that cause the majority of the damage to a particular crop. The data was compiled by crop and separated between field studies and interviews/ surveys. All data used for the analysis from the various sources are provided in Table 1 . Many studies provided a range of damage estimates for a single crop. If a minimum and maximum damage estimate was provided, these are listed in the "low" and "high" columns in the table. When only a single estimate was provided, the estimate was listed in the "mid" column. Therefore, the distinction between low, mid, and high was derived directly from the estimates provided by each specific data source and was not our interpretation of the relative size of the estimates.

To obtain a summary estimate of damage for each crop, two Monte Carlo simulations were performed. The first Monte Carlo simulation relied on data from field studies; the second on data 
from surveys and interviews. This distinction was made because of the difference in methodology between field studies and surveys and interviews. For the simulations, damage estimates from sources that provide a minimum and maximum damage were assumed to be uniformly distributed. Those studies that provide a minimum and maximum, as well as a most likely value, were assumed to have a triangular distribution where the most likely value is the mode. No distribution was assigned to studies that provide a single number; this number was assumed to occur with certainty. The simulation proceeds by randomly drawing a damage estimate from all of the individual estimates and assigning that estimate to some acre of land. This was done repeatedly for 1000 acres of land and the mean damage across those 1000 acres was calculated. To obtain a single summary result for each crop, the Monte Carlo simulation using field study data was given a $70 \%$ weight, while the other Monte Carlo simulation was given a $30 \%$ weight. This weighting assumed that field study data is more reliable than survey or interview data.

The expected yield loss per damaged acre was weighted by the fraction of planted acres of the crop that were affected by pests and then averaged (see Table 2). Because not all acres suffer damage, multiplying the percent yield loss per damaged acre by the percent of acres damaged gives the expected damage from bird and rodent pests for each crop. It should be noted that the method of weighting used here provides a conservative estimate of damages. Some studies that provided an estimate of the fraction of planted acres affected by a pest had already taken that into account when reporting yield loss. The yield loss estimates from such studies were used in the Monte Carlo simulation to derive the yield loss per acre affected by pest. Therefore, weighting this result again by the fraction of acres damaged puts a downward bias on the expected yield loss. However, failure to weight the results of the Monte Carlo simulation in this way would put upward bias on the damage estimates.

The extensive collection of damage estimates assembled allowed for a limited meta-analysis. Meta-analysis uses a statistical approach to review and summarize literature and previously obtained research results (Florax et al., 2002; Egger and Smith,

Table 2

Expected yield loss per damaged acre, percent of total acreage that suffers damage, and percent of total yield that is lost to bird and rodent pests.

\begin{tabular}{lcll}
\hline Crop & $\begin{array}{l}\text { Expected Yield } \\
\text { Loss Per Damaged } \\
\text { Acre (\%) }\end{array}$ & $\begin{array}{l}\text { Acres Damaged } \\
\text { (\% of total) }\end{array}$ & $\begin{array}{l}\text { Expected Damage } \\
\text { (\% yield loss) }\end{array}$ \\
\hline Almond & 5.1 & 50.8 & 2.6 \\
Artichoke & 11.8 & 70.0 & 8.3 \\
Broccoli & 9.5 & 42.1 & 4.0 \\
Carrots & 0.4 & 40.0 & 0.2 \\
Cherries & 11.1 & 34.0 & 3.8 \\
Citrus, oranges & 1.0 & 30.0 & 0.3 \\
Citrus, lemons & 3.5 & 30.0 & 1.1 \\
Grapes, table & 5.4 & 67.5 & 3.6 \\
Grapes, wine & 10.7 & 67.5 & 7.2 \\
Hay, alfalfa & 24.0 & 17.0 & 4.1 \\
Lettuce & 6.1 & 42.1 & 2.6 \\
Melons & 4.2 & 17.5 & 0.7 \\
Nursery, flower & 3.0 & 20.0 & 0.6 \\
Nursery, & 5.0 & 100.0 & 5.0 \\
$\quad$ container & & & \\
Peaches & 1.6 & 40.0 & 0.6 \\
Pistachios & 8.4 & 53.0 & 4.5 \\
Rice & 0.7 & 39.0 & 0.3 \\
Rice, wild & 5.4 & 93.0 & 5.0 \\
Spinach & 6.1 & 42.1 & 2.6 \\
Strawberry & 2.6 & 30.0 & 0.8 \\
Tomato & 0.8 & 30.0 & 0.2 \\
Walnut & 5.0 & 40.0 & 2.0 \\
\hline & & & \\
\hline
\end{tabular}

1997). One function of a meta-analysis is to determine how different research methods affect the results of the study (Stanley, 2001). The type of meta-analysis performed here is more specifically called a meta-regression analysis (Stanley and Jarrell, 1989). Given the purpose of our study is, in part, a synthesis of previous damage estimates, a meta-analysis can provide several useful insights. First, the impact of the source of the damage estimate (e.g. field study, interview) on the magnitude of the reported damage can be investigated. For example, experts may only become aware of damage when it is abnormally high, and the data from interviews with these experts may reflect this. Second, there may be a trend in damages over time. Perhaps damage has decreased over time as producers gain damage management experience, or conversely, regulations may have reduced the ability of producers to use certain control methods. In either case, the meta-analysis can examine any resulting trend in the data.

Our study included many different crops and these crops do not suffer equal damage. This necessitated controlling for the type of crop in the meta-analysis. Unfortunately, there was an insufficient number of damage estimates available for most crops to make this statistically feasible. We were therefore forced to limit our sample to the four crops with the most observations: almonds, grapes, alfalfa, and lettuce.

The model we used for the meta-analysis assumes the damage estimate depends on the year of the study or expert interview, the type of crop, and whether the damage estimate was obtained from an interview with an expert. The model can therefore be written as

$$
\begin{aligned}
\text { damage }_{i}= & \beta_{1}+\beta_{2}\left(\text { year }_{i}\right)+\beta_{3}\left(\text { almonds }_{i}\right)+\beta_{4}\left(\text { grapes }_{i}\right) \\
& +\beta_{5}\left(\text { alfalfa }_{i}\right)+\beta_{6}\left(\text { expert }_{i}\right)+u_{i}
\end{aligned}
$$

In this equation, $i$ indicates the particular damage estimate observation (e.g., a 5\% estimate of lettuce damage from an expert). The year variable represents the year that the study or expert interview was performed. The estimated coefficient $\hat{\beta}_{2}$ can be interpreted as the change in the expected damage estimate when the year is increased by one. Stated differently, as all other factors are held constant, a one year movement closer to the present will change the expected damage by $\hat{\beta}_{2}$

The crop variables (almonds, grapes, alfalfa) are dummy variables that equal one if an observation came from that particular crop and zero otherwise. To avoid perfect collinearity among the variables, no dummy variable is specified for lettuce (Greene, 2003). Therefore, the estimated coefficients $\left(\hat{\beta}_{3}, \hat{\beta}_{4}, \hat{\beta}_{5}\right)$ reflect how much expected damage to the other crops differ from expected damage to lettuce. Similarly, expert is a dummy variable that equals one if the observation came from an interview with an expert and zero otherwise. No dummy variable was specified for other types of data sources to avoid perfect collinearity. The coefficient $\hat{\beta}_{6}$ indicates the difference in expected damage when the damage estimate comes from an interview with an expert instead of some other data source.

The model was estimated using ordinary least squares (OLS) (Greene, 2003). However, to account for both heterskedasticity and autocorrelation, the Newey-West estimator was used to obtain the covariance matrix of the OLS estimator (Newey and West, 1987). This insures hypothesis tests on the estimated coefficients are statistically valid. Hypothesis tests on the estimated coefficients were the standard two-tailed $t$-tests (Greene, 2003).

\section{Results}

The results of the Monte Carlo simulation and weighting method are given in Table 2. Expected (the weighted mean) yield loss per damaged acre is the direct result of the Monte Carlo simulation, 
while expected damage is the weighted result. Assessment of relative pest damage should be based on the expected damage rather than the expected damage per damaged acre. These summary estimates may be interpreted as the expected crop damage given the range of estimates provided by the various sources. While it is tempting to simply take the mean of the individual estimates for each crop, such an approach may lead to a biased result because many of the estimates from individual sources are not a single number. Referring back to Table 1, it is clear that some estimates were reported as a range or with some value within that range being reported as most likely. The expected damage estimates do not reveal any obvious ranking of damages according to crop categories, but they do indicate considerable differences in damage among the crops. Average artichoke damage, for example, is $8.3 \%$, but average damage to carrot acreage is only $0.2 \%$.

The results of the meta-analysis are presented in Table 3 . The constant term (equal to one for all observations) accounts for the fact that there is no dummy variable for lettuce and non-expert interview data sources and serves as a baseline from which we can interpret the coefficients on the dummy variables that were included. Thus, the coefficient on expert (7.714) means that the expected damage estimate from an expert interview is approximately $7.7 \%$ higher than the expected damage estimate from some other data sources. The corresponding t-stat (2.16) and $p$-value (0.033) indicate that for this variable, the data source matters and expert interviews yield larger damage estimates than other sources.

The other key variable of interest was year. The estimate of the coefficient (0.008) was statistically significant at the $1 \%$ level ( $p$-value of 0.002). This can be interpreted as a small upward trend in the damage estimates over time. The coefficients on the cropspecific dummy variables can be interpreted as the difference in the expected damage to those crops relative to expected lettuce damage when all other factors are held constant. Thus, almonds are expected to suffer about 5\% less damage than lettuce, grapes about the same level of damage as lettuce, and alfalfa about $15 \%$ greater damage. The alfalfa coefficient indicated statistical significance at commonly accepted levels ( $p$-value of 0.009). Although the other two are not statistically significant at the $5 \%$ or $10 \%$ level, the estimates are the best indication of the relative difference in damages between these crops and lettuce.

\section{Discussion}

U.S. agricultural production plays a crucial role in the domestic and world economy. California leads the nation in the production of agricultural goods, producing roughly $\$ 38$ billion worth of agricultural commodities annually (CDFA, 2010). Damage to these crops can be severe and lead to impacts that extend beyond the producer, resulting in losses to the state and national economies. To successfully combat bird and rodent damage to crops, producers, agricultural extension experts, and researchers must have an understanding of the level of damage to these economically important crops.

This review and synthesis provides the most comprehensive and current compilation of bird and rodent damage to select California crops available from scientific literature and expert

Table 3

Meta-analysis results.

\begin{tabular}{lllll}
\hline Variable & Coefficient & Standard Error & $t$-statistic & $p$-value \\
\hline Constant & -6.008 & 6.318 & -0.951 & 0.343 \\
year & 0.008 & 0.002 & 3.161 & 0.002 \\
almonds & -5.486 & 4.321 & -1.270 & 0.206 \\
grapes & -0.971 & 4.300 & -0.226 & 0.822 \\
alfalfa & 15.559 & 5.871 & 2.650 & 0.009 \\
expert & 7.714 & 3.572 & 2.160 & 0.033 \\
\hline
\end{tabular}

knowledge. While numerous sources contain components of the data presented in this study, collating this data into one study allowed for a Monte Carlo analysis and a synthesis of the data that provided new information including the influence of the data source on damage estimates, trends in damage estimates over time, and how estimates differ among crops.

The magnitude of the results of the Monte Carlo simulation and an examination of the assembled damage estimates indicate that damage remains significant despite the use of a variety of methods (e.g. rodenticides and avicides, trapping, exclusion, and chemical aversion) to control bird and rodent pests. Crops such as artichokes, wine grapes, and wild rice were damaged heavily, and it is likely that the economic impact of this damage is very large. Thus, there are significant benefits to be had by developing and implementing more effective pest control methods. It is also possible that effective means of control are available but are too costly to use. Therefore, efforts to lower the costs of currently available methods are likely warranted. Estimates of damage used in our study were pestrelated primary damages to the final fruit, nut, grain, vegetable, nursery, or forest product. For example, primary bird damage to grapes occurs when the bird plucks whole fruit or pecks at the fruit resulting in decreased yield (Tobin, 1984). Pocket gophers may cause secondary damage by tunnelling near a grape vine, but this is not damage to the final fruit and is not reflected in our damage estimate. Inclusion of this secondary damage would increase the estimated damages to agricultural crops.

Unfortunately, the effect of bird and rodent pests on many of the crops has not been studied and documented sufficiently. Some damage estimates had limited availability of sources, and many were based on expert opinion rather than actual field studies. Additionally, it is also important to note that the nature of vertebrate pests has changed over time and invasive species have become an increasing concern. Our study provides a baseline from which future examinations of invasive species impacts to these crops can be measured.

In conclusion, our study indicates that damage to select California crops can be significant. The summary estimates calculated in the analysis are valuable because they condense the wide-ranging individual estimates into a single, perhaps more usable, estimate for each crop. This enables targeted and efficient application of current pest control methods and can serve as a guide for the development of new methods. There are numerous extensions to the analysis that could be fruitful areas for future research. First, it would be advantageous to translate the yield loss due to pests into economic damages. When yield is reduced, producer revenue falls, impacting the regional economy as well as the producer as less is spent on wages and other goods. Additionally, the results may be incorporated into a wider examination of the benefits and costs of pest control methods. Our results indicate remaining damages with current pest control methods. Given that the presence of pests necessitate the use of pest control, the remaining damages could be combined with the cost of the pest control to develop an estimate of the total negative impact of bird and rodent pests on California growers.

\section{Acknowledgements}

Funding by the California Department of Food and Agriculture, Vertebrate Pest Advisory Research Council is gratefully acknowledged. We also thank the many people who provided advice and/or contributed data used in this research.

\section{References}

Berge, A.J., Delwiche, M.J., Gorenzel, W.P., Salmon, T.P., 2007. Bird control in vineyards using alarm and distress calls. Am. J. Enol. Vitic. 58 (1), 135-143. 
Bolda, M.P., 2008. Cooperative Extension, Santa Cruz County. University of California. Interview, October 2008.

California Department of Food and Agriculture (CDFA), 1984. Survey of Agricultural Commissioners: bird damage to nut crops. Calif. Department Food Agric. unpublishedreport.

California Department of Food and Agriculture (CDFA), 2010. "California Production Statisics 2009-2010". www.cdfa.ca.gov/statistics accessed 7/2010.

Crabb, A.C., Salmon, T.P., Marsh, R.E., 1986. Bird problems in California pistachio production. Pro. Vert. Pest Conf. 12, 295-302.

Crase, F.T., Stone, C.P., DeHaven, R.W., Mott, D.F., 1976. Bird Damage to Grapes in the United States with Emphasis on California. United states Department of the Interior, Fish and Wildlife Service Special Scientific Report - Wildlife No. 197. Washington, D.C.

Cummings, J.L., Shwiff, S.A., Tupper, S.K., 2005. Economic impacts of blackbird damage to the rice industry. Pro. Wildl. Damage Manag. Conf. 11, 317-322.

DeHaven, R.W., 1971. Blackbirds and the California rice crop. Rice J. 74 (8), 7-14.

DeHaven, R.W., 1974. Bird damage to seeds and seedling crops in California, a questionnaire survey. Technical report No. 11, Work Unit DF-102.3. Denver wildlife research Center.

DeHaven, R.W., Mott, D.F., Guarino, J.L., Besser, J.F., Knittle, C.E., Schafer Jr., E.W. 1979. Methiocarb for repelling birds from ripening sweet cherries. Int. Pest Control, 12-14. January-February 1979.

DeHaven, R.W., Hothem, R.L., 1980. Estimating bird damage from damage incidence in wine grape vineyards. Am. J. Enol. Vitic. 32, 1-4.

Delwiche, M.J., Houk, A., Gorenzel, W.P., Salmon, T.P., 2007. Control of crows in almonds by broadcast distress calls. Trans. ASABE 50, 675-682.

Egger, M., Smith, G.D., 1997. Meta-analysis: potentials and promise. Br. Med. J. 315, 1371-1374.

Emlen, J.T., 1937. Bird damage to almonds in California. The Condor 39 (5), 192-197.

Florax, R.J.G.M., de Groot, H.L.F., de Mooij, R.A., 2002. Meta-analysis: a Tool for Upgrading Inputs of Macroeconomic Policy Models. Discussion Paper, TI 2002041/3. Tinbergen Institute. http://www.tinbergen.nl/ti-publications/discussionpapers.php?paper $=272$ accessed 5/2010.

Gadd Jr., P., 1996. Use of the modified Australian crow trap for the control of depredating birds in Sonoma County. Pro. Vert. Pest Conf. 17, 103-107.

Goymerac, E., 2008. Wildlife Services Operations Napa County, Interview, October 2008

Greene, W.H., 2003. Econometric Analysis, Fifth ed. Prentice Hall, Upper Saddle River, New Jersey.

Hasey, J., Salmon, T.P., 1993. Crow damage to almonds increasing; no foolproof solution in sight. Calif. Agric., 21-23. September-October 1993.

Hothem, R.L., Mott, D.F., DeHaven, R.W., Guarino, J.L., 1981. Mesurol as a bird repellent on wine grapes in Oregon and California. Am. J. Enol. Vitic 32, 150-154.

Hueth, B.M., Cohen, D., Sangrujee, N., Zilberman, D., 1997. Economic Impact of Nonpredator Vertebrate Pest Damage in California Agriculture. Final Report Prepared for the California Department of Food and Agriculture, Vertebrate Pest Control Research Advisory Council, Sacramento, CA.

Kalinowski, S.A., deCalesta, D.S., 1981. Baiting regimes for reducing ground squirrel damage to alfalfa. Wildl. Soc. Bull. 9, 268-272.

Marcum, D.B., Gorenzel, W.P., 1994. Grower practices for blackbird control in wild rice in California. Pro. Vert. Pest Conf. 16, 243-249.

Marsh, R., 1998. Historical review of ground squirrel crop damage in California. Int. Biodeterior. Biodegradation 42, 93-99.

Marsh, R., 2008. Professor Emeritus, University of California, Davis, Interview, October 2008.

Maya, J., 2008. Lettuce grower, Fresno County, Interviewed by the office of Fred Rinder, October 2008.

Moinar, R., 2008. Extension Specialist, Fresno County. University of California, Interview. October 2008

Muramoto, J., Gliessman, S.R., Shennan, C., 2005. Project report submitted to the Organic Farming Research Foundation: maintaining agroecosystem health in an organic strawberry/vegetable rotation system final project report.
National Agricultural Statistics Service (NASS), 1999. Fruit Wildlife Damage. Agricultural Statistics Board. U.S. Department of Agriculture.

National Agricultural Statistics Service (NASS), 2002. U.S. Wildlife Damage. Agricultural Statistics Board. U.S. Department of Agriculture.

Newey, W., West, K., 1987. A Simple Positive Semi-Definite, Heteroskedasticity and autocorrelation Consistent covariance matrix. Econometrica 55, 703-708.

Pearson, A.B., Gorenzel, W.P., Salmon, T.P., 2000. Lesser-know vertebrate pests of almonds in California. Pro. Vert. Pest Conf. 19, 365-376.

Pitts, G., 2008. Table Grape Grower, Fresno County Interview by the Office of Fred Rinder, October 2008

Razee, D., 1976. California agriculture loses $\$ 40$ million a year from Vertebrate pest damage. Calif. Farmer, 60-61. April 17, 1976.

Roach, B., 2008. Deputy Agricultural Commissioner, Monterey County Interview October 2008.

Sagardia, B., Sagardia, M., 2008. Almond Growers, Fresno County Interview, October 2008.

Salmon, T.P., Gorenzel, W.P., Ford, W.K., 1997. Test of a Taped Alarm Call to Reduce Crop Damage in Almonds. Final report prepared for the California Department of Food and agriculture. Vertebrate Pest Control Research Advisory Council. Sacramento, CA. Contract No. 96-0486.

Salmon, T.P., Gorenzel, W.P., Pearson, A.B., Ryan, S.R., 1999. A test of broadcast calls to reduce crow damage in almonds. Final report prepared for the California Department of Food and agriculture. Vertebrate Pest Control Research Advisory Council. Sacramento, CA. Contract No. 96-0486-II.

Salmon, T.P., Gorenzel, W.P., Pearson, A.B., 2000. An operational crow contro program using broadcast calls. Final report prepared for the California Department of Food and Agriculture. Vertebrate Pest Control Research Advisory Council. Sacramento, CA. Contract No. 96-0486-III.

Sauer, W.C., 1984. Impact of the Belding's Ground Squirrel, Spermophilus beldingi, on alfalfa production in northeastern California. Pro. Vert. Pest Conf. 11, $20-23$.

Sexton, S.E., Lei, Z., Zilberman, D., 2007. The economics of pesticides and pest control. Int. Rev. Environ. Resource Econ. 1, 271-326.

Simpson, G., 1972. Some approaches to controlling depredations by crows and jays in Tulare County. Proc. Vertebrate Pest Conf. 5, 112-117.

Stanley, T.D., Jarrell, S.B., 1989. Meta-Regression analysis: a quantitative method of literature surveys. J. Econ. Surv. 3 (2), 161-170.

Stanley, T.D., 2001. Wheat from chaff: meta-analysis as quantitative literature review. J. Econ. Perspect. 15 (3), 131-150.

Stone, C.P., 1973. Bird damage to agricultural crops in the United States - a current summary. Bird Control Semin. Proc., 263-267.

Strmiska, M., 2008. Pest Control Advisor, Fresno County Interview by the Office of Fred Rinder, October 2008.

Taber, M., 2008. Wildlife Control Technology, Fresno Interview, October 2008.

Tobin, M.E., 1984. Relative grape damaging potential of three species of birds California Agriculture, March-April 1984, pp. 9-10.

Vasquez, S.J., 2008. Cooperative Extension, Fresno County University of California Interview, October 2008

Whisson, D.A., Orloff, S.B., Lancaster, D.L., 1999. Alfalfa yield loss from Belding's ground squirrels in northeastern California. Wildl. Soc. Bull. 27, 178-183.

Whisson, D.A., Orloff, S.B., Lancaster, D.L., 2000. The economics of managing Belding's ground squirrels in alfalfa in northeastern California. Human conflicts with wildlife: economic considerations. Proc. USDA Natl. Wildl. Res. Cent. Symposia. pp. 104-108.

Wilen, C., 2008. Cooperative Extension, San Diego, Orange, and Los Angeles Counties University of California, Interview, October 2008.

Witmer, D., 2008. Napa County Agricultural Commissioner Interview, October 2008.

York, D.L., Cummings, J.L., Engeman, R.M., Davis Jr., J.E., 2000. Evaluation of Flight Control $^{\mathrm{TM}}$ and Mesurol $\odot$ as repellents to reduce horned lark (Eremophila alpestris) damage to lettuce seedlings. Crop Prot. 19, 201-203. 